\title{
Error Analysis of Some Techniques for Updating Orthogonal Decompositions
}

\begin{abstract}
By C. C. Paige*
Abstract. We consider accurate and efficient methods for updating the result of the transformation $C=B Q, Q$ orthogonal, of a given matrix $B$ when $Q$ is available. Adding or deleting a row, or adding a column of $B$ leads to a continuation of the original transformation, and as such is numerically stable. In particular, we discuss a wellknown method for updating when a column of $B$ is deleted, and show that it is as numerically stable as the problem allows. The results extend to two-sided transformations of the form $C=Z^{T} B Q$. The methods and analyses are independent of the form or rank of $B$ and $C$, and so are widely applicable.
\end{abstract}

1. Introduction. In many problems we are interested in computing the orthogonal transformation

$$
C=B Q
$$

of a given $m \times n$ matrix $B$ for a certain orthogonal matrix $Q$. Often $Q$ will be chosen so that $C$ has a certain form, such as $(L, 0)$ where $L$ is lower trapezoidal of full column rank. However, the results will be more useful if they are given for a general orthogonal transformation in (1.1). We will be interested in updating $C$ and $Q$ in an efficient and numerically stable way when a column or row of $B$ is added or deleted. In order to do this we will assume $Q$ is available whenever it is needed, and we will not discuss the less reliable methods that use $C$ to find information on $Q$ instead of using $Q$ itself, even though they are usually faster. The problem is easily solved when a row is appended to or deleted from $B$, and when a column is appended to $B$. Most of this note will treat the deletion of a column from $B$.

Here capital italic letters will denote matrices, lower case italic will denote vectors and indices, and greek will denote scalars, except $\delta B$, etc., will denote a matrix of small elements. Superscript $T$ will denote transpose, and we will use the 2-norm throughout. In the rounding error analysis the indexed scalars $\epsilon_{i}$ will denote nonnegative numbers bounded above by the product of the floating-point computer precision $\epsilon$ and small constants dependent only on the number of transformations and possibly the dimensions of the problems.

It follows from the work of Wilkinson [4] that if $B$ is given and $Q$ is the product

Received September 27, 1977.

AMS (MOS) subject classifications (1970). Primary 65F25, 65 G05.

Key words and phrases. Error analysis, updating techniques, orthogonal decompositions, orthogonal matrices.

*Supported by National Research Council of Canada grant A8652. 
of numerically stable elementary hermitians or plane rotations, then the computed matrices $C$ and $Q$ in (1.1) satisfy

$$
\begin{gathered}
C=(B+\delta B) \bar{Q}, \\
\|Q-\bar{Q}\|=\epsilon_{0}, \\
\left\|\delta b_{j}\right\|=\epsilon_{j}^{\prime}\left\|b_{j}\right\|,
\end{gathered}
$$

where $\delta b_{j}$ and $b_{j}$ are the columns of $\delta B^{T}$ and $B^{T}$, respectively, and $\bar{Q}$ is orthogonal. Because of this result the transformation is numerically stable, and we will be interested in updating techniques which have this same form of numerical stability, or as close to it as we can get.

If additional orthogonal transformations are applied from the left, or right, or both, so that ideally

$$
C=Z^{T} B Q
$$

then it is straightforward, see for example [3, Section 6], to show that the computed $C, Z$, and $Q$ satisfy

$$
\begin{gathered}
C=\bar{Z}^{T}(B+\delta B) \bar{Q}, \\
\|Q-\bar{Q}\|=\epsilon_{1}, \quad\|Z-\bar{Z}\|=\epsilon_{2}, \\
\|\delta B\|=\epsilon_{3}\|B\|,
\end{gathered}
$$

where $\bar{Z}$ and $\bar{Q}$ are orthogonal. Here $\bar{Z}$ combines the rows of $B$, and so (1.8) is weaker than (1.4).

2. Updating Techniques. Many effective updating techniques have been developed over the last few years, and [1] and [2] are good sources for the techniques that will be mentioned here, along with many others. The purpose here is to be reasonably brief, but give a general analysis of techniques based on the use of $Q$ for updating (1.1).

In what follows

$$
\widetilde{C}=\widetilde{B} \widetilde{Q}
$$

will be the theoretical result brought about by updating the transformation (1.1) when a row $b^{T}$ or a column $b$ is appended to or deleted from $B$. We will describe all techniques in their simplest forms; other computations will just be straightforward extensions of these.

(a) Append a row $b^{T}$ to $B$. Compute

$$
c^{T}=b^{T} Q
$$

then $\widetilde{Q}=Q$ and the transformation

$$
\widetilde{C}=\left(\begin{array}{c}
C \\
c^{T}
\end{array}\right)=\left(\begin{array}{c}
B \\
b^{T}
\end{array}\right) Q=\widetilde{B} \widetilde{Q}
$$

is already available. 
(b) Delete a row $b^{T}$ from $B=\left(\begin{array}{c}\widetilde{B} \\ b\end{array}\right)$. Write

$$
C=\left(\begin{array}{c}
\widetilde{C} \\
c^{T}
\end{array}\right)=B Q=\left(\begin{array}{c}
\widetilde{B} \\
b^{T}
\end{array}\right) Q,
$$

so with $\widetilde{Q}=Q$, the transformation is updated by deleting the last row of $C$.

(c) Append a column $b$ to $B$. Write

$$
\widetilde{C}=(C, b)=(B, b)\left(\begin{array}{ll}
Q & 0 \\
0 & 1
\end{array}\right)=\widetilde{B} \widetilde{Q}
$$

(d) Delete a column $b$ from $B=(\widetilde{B}, b)$. Apply an orthogonal matrix $P$ to the last row of $Q$, so that if $e_{n}$ is the last column of the $n \times n$ unit matrix $I$,

$$
\left(\begin{array}{c}
C \\
e_{n}^{T} Q
\end{array}\right) P=\left(\begin{array}{cc}
\widetilde{C} & c \\
0 & \pm 1
\end{array}\right)
$$

It follows that $Q P$ has the form

$$
Q P=\left(\begin{array}{cc}
\widetilde{Q} & 0 \\
0 & \pm 1
\end{array}\right)=N \text { say }
$$

Clearly, $\widetilde{Q}$ is orthogonal and

$$
(\widetilde{C}, c)=C P=B Q P=(\widetilde{B} \widetilde{Q}, \pm b),
$$

so deleting the last column gives the required new decomposition.

We note that (a) and (d) require $Q$ to carry out the update, while (b) and (c) require no computation. $Q$ is unaltered in (a) and (b), and trivially modified in (c), but (d) requires a nontrivial transformation of $Q$ and depends on a nontrivial theoretical result. The extensions of these methods to appending or deleting other than the last row or column of $B$, and to placing the \pm 1 elsewhere in the last row in (2.6), are trivial, but require more complicated notation and so will be ignored. The results of the analyses will clearly hold for these extensions. The methods and analyses are independent of the form and rank of $B$ or $C$, and the particular orthogonal matrices $Q$ and $P$ used to give (1.1) and (2.6), and so are quite general.

On examination it can be seen that the techniques in (a), (b) and (c) are just extensions of (1.1), and so the results (1.2) to (1.4) hold; and the updating techniques are numerically stable, giving bounds exactly as if the computation had started with $\widetilde{B}$ instead of $B$. In Section 4 we will show that (d) is as numerically stable as can be expected for the problem, giving a rounding error result with similar form to (1.2) and (1.4). In particular computations $C$ will often have a specific form, and we will want $\widetilde{C}$ to have the same form. In such cases (a), (b), (c) and (d) will be designed so this form can be efficiently regained by applying more numerically stable transformations. If this is done by applying orthogonal transformations from the right to the relevant parts of (2.3) to (2.5), and (2.8), then these will just be further continuations of (1.1) and as such will make the computations numerically stable, or in (d) as numerically stable as can be expected for the problem. 
3. A Property of Orthogonal Matrices. In order to prove the numerical stability of column deletion in (1.1), we will use the following general property of orthogonal matrices. Let

$$
\left(\begin{array}{cc}
G & g \\
h^{T} & \gamma
\end{array}\right)
$$

be a partitioned orthogonal matrix. If $\gamma= \pm 1$ then $g=h=0$ and $G$ is orthogonal; otherwise

$$
\bar{G} \equiv G+\alpha g h^{T}
$$

is orthogonal when $\alpha=-(\gamma-1)^{-1}$ or $-(\gamma+1)^{-1}$, and then

$$
\bar{G} h= \pm g, \quad g^{T} \bar{G}= \pm h^{T} .
$$

This can be shown as follows. From the orthogonality of (3.1)

$$
\begin{gathered}
G h+\gamma g=0, \quad G^{T} g+\gamma h=0, \\
G G^{T}+g g^{T}=I, \quad g^{T} g+\gamma^{2}=h^{T} h+\gamma^{2}=1,
\end{gathered}
$$

so

$$
\overline{G G}^{T}=I-\left[1+2 \alpha \gamma-\alpha^{2}\left(1-\gamma^{2}\right)\right] g g^{T}=I
$$

for the given values of $\alpha$. Next

$$
\begin{gathered}
\bar{G} h=G h+\alpha h^{T} h g=\left(\alpha h^{T} h-\gamma\right) g=\left[\alpha\left(1-\gamma^{2}\right)-\gamma\right] g= \pm g, \\
\bar{G}^{T} g=\left(\alpha g^{T} g-\gamma\right) h= \pm h .
\end{gathered}
$$

These results will be useful to us when $\|g\|$ is small.

4. Analysis of Column Deletion. To analyse the computations required for (d) in Section 2, we assume that the computed $C$ and $Q$ satisfy (1.2) to (1.4). The computations described by (2.6) and (2.7) will then give computed $\widetilde{C}, c$, and $N$ satisfying

$$
\begin{gathered}
\left(\begin{array}{c}
C+\delta C \\
e_{n}^{T} \bar{Q}+\delta q^{T}
\end{array}\right) \bar{P}=\left(\begin{array}{cc}
\widetilde{C} & c \\
0 & \pm 1
\end{array}\right), \\
\|\delta q\|=\epsilon_{4}, \quad\|N-\overline{Q P}\|=\epsilon_{5}, \\
\left\|\delta c_{j}\right\|=\epsilon_{j}^{\prime \prime}\left\|b_{j}\right\|,
\end{gathered}
$$

where $\delta c_{j}$ are the columns of $\delta C^{T}$, and $\bar{P}$ and so $\overline{Q P}$ is orthogonal. In order to use the result of Section 3 we write

$$
\overline{Q P}=\left(\begin{array}{cc}
G & g \\
h^{T} & \gamma
\end{array}\right),
$$

where from (4.1) and (4.2)

$$
h^{T} h+(\gamma \mp 1)^{2}=\epsilon_{4}^{2} .
$$


This combines with (3.5) to give

$$
\begin{gathered}
1 \mp \gamma=\epsilon_{4}^{2} / 2, \\
h^{T} h=g^{T} g=\epsilon_{4}^{2}\left(1-\epsilon_{4}^{2} / 4\right) .
\end{gathered}
$$

If $\gamma \sim 1$, we take $\alpha=-(\gamma+1)^{-1}$ in Section 3; otherwise, $\gamma \sim-1$ and we take $\alpha=$ $-(\gamma-1)^{-1}$, but in either case we have an orthogonal matrix

$$
\begin{gathered}
\bar{G} \equiv G+\alpha g h^{T}, \\
\left\|\alpha g h^{T}\right\|=|\alpha| h^{T} h=\epsilon_{4}^{2} / 2 .
\end{gathered}
$$

This shows that the leading part of $\overline{Q P}$ is within $\epsilon_{4}^{2} / 2$ of an orthogonal matrix, which is a better result than might initially have been expected. From (4.2) we see that the leading part $\widetilde{Q}$ of $N$ satisfies

$$
\|\widetilde{Q}-\bar{G}\|=\|\widetilde{Q}-G+G-\bar{G}\| \leqslant \epsilon_{5}+\epsilon_{4}^{2} / 2,
$$

and so our computed result $\widetilde{Q}$ is close to an orthogonal matrix. Next from (1.2) and (4.1)

$$
\left(B+\delta B+\delta C \bar{Q}^{T}\right) \overline{Q P}=(\widetilde{C}, c)
$$

or if we write $\delta B+\delta C \bar{Q}^{T}=\left(\delta \widetilde{B}^{\prime}, \delta b\right)$ and use (4.4) and (4.8),

$$
\begin{aligned}
\widetilde{C} & =\left(\widetilde{B}+\delta \widetilde{B}^{\prime}\right) G+(b+\delta b) h^{T} \\
& =\left(\widetilde{B}+\delta \widetilde{B}^{\prime}\right)\left(\bar{G}-\alpha g h^{T}\right)+(b+\delta b) h^{T} \\
& =(\widetilde{B}+\delta \widetilde{B}) \bar{G}
\end{aligned}
$$

which has the form of (1.2). From (1.4), (4.3), (4.7) and (4.9)

$$
\begin{gathered}
\delta \widetilde{B} \bar{G}=\left(\delta \widetilde{B}^{\prime}, \delta b\right)\left(\begin{array}{c}
G \\
h^{T}
\end{array}\right)+(\widetilde{B}, b)\left(\begin{array}{c}
-\alpha g \\
1
\end{array}\right) h^{T}, \\
\left\|\delta \widetilde{b}_{j}\right\| \leqslant\left(\epsilon_{j}+\epsilon_{j}^{\prime \prime}+\epsilon_{4}\right)\left\|b_{j}\right\|,
\end{gathered}
$$

where $\delta \widetilde{b}_{j}$ is the $j$ th column of $\delta \widetilde{B}^{T}$.

We see that (4.12) and (4.10) have exactly the same form as (1.2) and (1.3), and (4.13) has similar form to (1.4), different rows of $B$ having no effect on each other. However, the bound (4.13) depends on $B=(\widetilde{B}, b)$ rather than just $\widetilde{B}$; and if $\|b\|>>$ $\|\widetilde{B}\|$, the new decomposition $\widetilde{C}=\widetilde{B} \widetilde{Q}$ might not be as accurate as if it had been computed directly from $\widetilde{B}$. However, (4.13) shows that if some elements of $b$ are reasonable, the corresponding rows of $\widetilde{C}$ will be as accurate as if they had initially come from $\widetilde{B}$. The updating difficulty with large elements of $b$ is inherent in the problem, and is the equivalent of cancellation for scalars. Suppose $|\beta|>>|\alpha|,|\gamma|$, and we form $\zeta:=$ $\beta+\alpha ; \eta:=\zeta+\gamma ; \mu:=\eta-\beta$; on a floating-point computer. Then $\mu$ will probably have a large relative error as an approximation to $\alpha+\gamma$. Nevertheless, we have shown that method (d) in Section 2 is as good as is possible for the problem. 
A final reassuring feature of the algorithm is that in (4.11), using (4.6), (4.7), (1.4) and (4.3),

$$
\begin{aligned}
c & =\left(\widetilde{B}+\delta \widetilde{B}^{\prime}\right) g+\gamma(b+\delta b) \\
& = \pm b+B\left(\begin{array}{c}
g \\
\mp \epsilon_{4}^{2} / 2
\end{array}\right)+\left(\delta B+\delta C \bar{Q}^{T}\right)\left(\begin{array}{l}
g \\
\gamma
\end{array}\right) \\
& = \pm b+\delta c
\end{aligned}
$$

where $\delta \gamma_{j}$ is the $j$ th element of $\delta c$ and $b_{j}^{T}$ is the $j$ th row of $B=(\widetilde{B}, b)$. So we see the computed $c$ will be very accurate, unless $\|b\|>>\widetilde{B} \|$.

5. Comments and Extension to $C=Z^{T} B Q$. We have shown that if we have an orthogonal decomposition (1.1) and we update it using any combination of (a), (b), and (c) in Section 2, then the result (1.2) to (1.4) will hold for our final matrices $B, C$ and $Q$. If we also include (d) in the combination, then $\left\|b_{j}\right\|$ in (1.4) must be the largest such norm encountered for the $j$ th column of $B^{T}$ during the computations. These results are as good as can be hoped for the problem; and in particular, the most complicated computation (d) was seen to have some very nice properties. These results hold no matter what stable transformations of the form (1.1) are carried out in between the updating steps.

If we now consider updating (1.5), we see that whether a row or a column is appended to $B$, the updating technique and its analysis will effectively be the same. For example, with $Q$ available,

$$
\widetilde{C}=\left(\begin{array}{c}
C \\
c^{T}
\end{array}\right)=\left(\begin{array}{c}
Z^{T} B Q \\
b^{T} Q
\end{array}\right)=\left(\begin{array}{cc}
Z^{T} & 0 \\
0 & 1
\end{array}\right)\left(\begin{array}{c}
B \\
b^{T}
\end{array}\right) Q=\widetilde{Z}^{T} \widetilde{B} \widetilde{Q}
$$

which is a continuation of (1.5), and so the computational results satisfy the equivalent of (1.6) to (1.8). Also, deleting a row from $B$ in (1.5) is effectively the same as deleting a column; but these involve a computation of the form (d) in Section 2 . For example, with $Q$ available we note that deleting the column $b$ from $B=(\widetilde{B}, b)$ is the same as deleting the column $Z^{T} b$ from $Z^{T} B=\left(Z^{T} \widetilde{B}, Z^{T} b\right)$, so Section 2 (d) is identical except that (2.8) becomes, with $\widetilde{Z} \equiv Z$,

$$
(\widetilde{C}, c)=C P=Z^{T} B Q P=\left(\widetilde{Z}^{T} \widetilde{B} \widetilde{Q}, \pm Z^{T} b\right) .
$$

The analysis of this is identical to that in Section 4, except for (4.3), which becomes

$$
\|\delta C\|=\epsilon_{6}\|B\|,
$$

and in (4.11) and the following, $B$ and $\delta B$ are replaced by $\bar{Z}^{T} B$ and $\bar{Z}^{T} \delta B$ to give

$$
\begin{gathered}
\widetilde{C}=\bar{Z}^{T}(\widetilde{B}+\delta \widetilde{B}) \bar{G}, \\
\|\delta \widetilde{B}\|=\epsilon_{7}\|B\|,
\end{gathered}
$$

so, with (1.7) and (4.10) still holding, the bounds are similar to those of (1.6) to (1.8). 
We see that (1.5) can be repeatedly updated, and orthogonal transformations can be applied to the left and right to give $\widetilde{C}$ any desired form, and the results (1.6) to (1.8) will still hold at any stage of the computation, except that if deletion occurs at any stage, then $\|B\|$ in (1.8) must be the largest such norm encountered. This extension is important for proving the numerical stability of some updating techniques for some methods that solve the generalized least squares problem [3].

School of Computer Science

McGill University

805 Sherbrooke Street W.

Montreal, Quebec, Canada

1. P. E. GILL, G. H. COLUB, W. MURRAY \& M. A. SAUNDERS, "Methods for modifying matrix factorizations," Math. Comp., v. 28, 1974, pp. 505-535.

2. C. L. LAWSON \& R. J. HANSON, Solving Least Squares Problems, Prentice-Hall, Englewood Cliffs, N. J., 1974.

3. C. C. PAIGE, "Computer solution and perturbation analysis of generalized linear least squares problems," Math. Comp., v. 33, 1979, pp. 171-183.

4. J. H. WILKINSON, The Algebraic Eigenvalue Problem, Clarendon Press, Oxford, 1965. MR 32 \#1894. 